\title{
NORMAL FAMILIES OF MEROMORPHIC FUNCTIONS WHOSE POLES ARE LOCALLY UNIFORMLY DISCRETE
}

\author{
XIAO-YI LIU
}

\begin{abstract}
Let $h$ be a positive number, and let $a(z)$ be a function holomorphic and zerofree on a domain $D$. Let $\mathscr{F}$ be a family of meromorphic functions on $D$ such that for every $f \in \mathscr{F}, f(z)=0 \Rightarrow f^{\prime}(z)=a(z)$ and $f^{\prime}(z)=a(z) \Rightarrow\left|f^{\prime \prime}(z)\right| \leq h$. Suppose that each pair of functions $f$ and $g$ in $\mathscr{F}$ have the same poles. Then $\mathscr{F}$ is normal on $D$.
\end{abstract}

\section{Introduction}

A family $\mathscr{F}$ of meromorphic functions on a plane domain $D \subset \mathbb{C}$ is said to be normal on $D$ in the sense of Montel if each sequence $\left\{f_{n}\right\} \in \mathscr{F}$ contains a subsequence which converges spherically uniformly on each compact subset of $D$. See [4], [7], [9].

For two functions $f$ and $g$ meromorphic on $D$ in $\mathbb{C}$, and two complex numbers or meromorphic functions $a$ and $b$, we write $f(z)=a(z) \Rightarrow g(z)=b(z)$ if $g(z)=b(z)$ whenever $f(z)=$ $a(z)$, and write $f(z)=a(z) \Leftrightarrow g(z)=b(z)$ if $f(z)=a(z)$ if and only if $g(z)=b(z)$. When $a$ is a complex value and $f(z)=a \Leftrightarrow g(z)=a$, we also say that $f$ and $g$ share the value $a$ or $a$ is a shared value of $f$ and $g$. For families of meromorphic functions, the connection between normality and shared values has been studied frequently following Schwick's initial paper [8].

The starting point of this paper is the following result.

Theorem 1.1 ([1] Theorem 4). Let $h$ be a positive number, $k \geq 2$ be an integer, and let $a(z)$ be a function which is holomorphic and zero-free on $D$. Then the family $\mathscr{F}=\{f\}$ of holomorphic functions on D such that $f(z)=0 \Rightarrow f^{\prime}(z)=a(z)$ and $f^{\prime}(z)=a(z) \Rightarrow\left|f^{(k)}(z)\right| \leq h$ is normal on $D$.

We remark that with the same notations, the family $\mathscr{F}=\{f\}$ of meromorphic functions on $D$ such that $f(z)=0 \Rightarrow f^{\prime}(z)=a(z)$ and $f^{\prime}(z)=a(z) \Rightarrow\left|f^{(k)}(z)\right| \leq h$ is not normal in general (at least for $k=2$ ) even if $a(z)$ is a nonzero constant. This is shown by Example 1 in [2] where

Received November 7, 2011, accepted August 8, 2013.

2010 Mathematics Subject Classification. 30D45.

Key words and phrases. Meromorphic functions, normal families, shared values.

The work is supported by National Natural Science Foundation of China (Grant NO. 11171045). 
it is proved that such a family $\mathscr{F}$ is normal if the poles of each function in $\mathscr{F}$ have sufficiently large multiplicities. We give here another condition on poles that enables the family $\mathscr{F}$ to be normal. In the sequel, we say that the poles of functions in $\mathscr{F}$ are locally uniformly discrete on $D$, if for each point $z_{0} \in D$, there exists $\delta=\delta\left(z_{0}\right)>0$ such that each function $f \in \mathscr{F}$ has at most one pole (ignoring multiplicity) in the disk $\Delta\left(z_{0}, \delta\right)=\left\{z:\left|z-z_{0}\right|<\delta\right\} \subset D$.

Theorem 1.2. Let $h$ be a positive number and $a(z)$ be a function which is holomorphic and zero-free on $D$. Then the family $\mathscr{F}=\{f\}$ of meromorphic functions on $D$ such that $f(z)=0 \Rightarrow$ $f^{\prime}(z)=a(z)$ and $f^{\prime}(z)=a(z) \Rightarrow\left|f^{\prime \prime}(z)\right| \leq h$ is normal on $D$, provided that the poles of functions in $\mathscr{F}$ are locally uniformly discrete on $D$.

Notice that if each pair of functions $f$ and $g$ in $\mathscr{F}$ have the same poles (or share the value $\infty$ ) on $D$, then the poles of functions in $\mathscr{F}$ are locally uniformly discrete in $D$. So we have the following corollary to Theorem 1.2.

Theorem 1.3. Let $h$ be a positive number and $a(z)$ be a function which is holomorphic and zero-free on $D$. Then the family $\mathscr{F}=\{f\}$ of meromorphic functions on $D$ such that $f(z)=0 \Rightarrow$ $f^{\prime}(z)=a(z)$ and $f^{\prime}(z)=a(z) \Rightarrow\left|f^{\prime \prime}(z)\right| \leq h$ is normal on $D$, provided that each pair of functions $f$ and $g$ in $\mathscr{F}$ have the same poles on $D$.

\section{Some lammas}

In order to prove our theorems, we require the following results. We assume the standard notation of value distribution theory, as presented and used in [4]. The first lemma is a special case of [6, Lemma 2].

Lemma 2.1. Let $\mathscr{F}$ be a family of functions meromorphic on D. Suppose that there exists $A \geq 1$ such that $f(z)=0 \Rightarrow\left|f^{\prime}(z)\right| \leq A$ for each $f \in \mathscr{F}$. If $\mathscr{F}$ is not normal at some point $z_{0} \in D$, there exist points $z_{n} \in D$ with $z_{n} \rightarrow z_{0}$, positive numbers $\rho_{n} \rightarrow 0$ and functions $f_{n} \in \mathscr{F}$ such that the sequence $g_{n}(\zeta)=\rho_{n}^{-1} f_{n}\left(z_{n}+\rho_{n} \zeta\right)$ converges spherically locally uniformly on $\mathbb{C}$ to a nonconstant meromorphic function $g$ which is of finite order and satisfies $g^{\sharp}(\zeta) \leq g^{\sharp}(0)=A+1$.

Here, as usual, $g^{\sharp}(\zeta)=\left|g^{\prime}(\zeta)\right| /\left(1+|g(\zeta)|^{2}\right)$ is the spherical derivative.

Lemma 2.2 ([5]). Let $f$ be a meromorphic function on $\mathbb{C}$ of finite order. Then for each positive integer $k$,

$$
m\left(r, \frac{f^{(k)}}{f}\right)=O(\log r), r \rightarrow \infty .
$$

Lemma 2.3 ([4] Corollary to Theorem 3.5). Let $f$ be a transcendental meromorphic function, and let a be a non-zero value. Then, for each positive integer $k$, either $f$ or $f^{(k)}-$ a has infinitely many zeros. 
Lemma 2.4 ([2] Remark, [1] Lemma 6). Let g be a nonconstant entire function, $k \geq 2$ be an integer, and let a be a nonzero finite value. If $g(z)=0 \Rightarrow g^{\prime}(z)=a$ and $g^{\prime}(z)=a \Rightarrow g^{(k)}(z)=0$, then $g(z)=a\left(z-z_{0}\right)$, where $z_{0}$ is a constant.

Lemma 2.5. Let $g$ be a function meromorphic on $\mathbb{C}$ of finite order and with finitely many poles, and let a be a nonzero finite value. If $g(z)=0 \Rightarrow g^{\prime}(z)=a$ and $g^{\prime}(z)=a \Rightarrow g^{\prime \prime}(z)=0$, then $g$ is a rational function.

Proof. Suppose that $g$ is transcendental, then by Lemma 2.4, $g$ is not entire and by the assumption, $N(r, g)=O(\log r)$. Since $g(z)=0 \Rightarrow g^{\prime}(z)=a$ and $g^{\prime}(z)=a \Rightarrow g^{\prime \prime}(z)=0$, the zeros of $g$ are simple and $g(z)=0 \Rightarrow g^{\prime \prime}(z)=0$. It follows that

$$
N\left(r, \frac{g^{\prime \prime}}{g}\right) \leq 2 N(r, g)=O(\log r) .
$$

On the other hand, as $g$ is of finite order, by Lemma 2.2, we have

$$
m\left(r, \frac{g^{\prime \prime}}{g}\right)=O(\log r) .
$$

Hence

$$
T\left(r, \frac{g^{\prime \prime}}{g}\right)=m\left(r, \frac{g^{\prime \prime}}{g}\right)+N\left(r, \frac{g^{\prime \prime}}{g}\right)=O(\log r) .
$$

This shows that the function

$$
R=\frac{g^{\prime \prime}}{g}
$$

is a rational function. Since $g$ is not entire, $R$ is nonconstant and has at least one (double) pole. Thus by the condition $g(z)=0 \Rightarrow g^{\prime}(z)=a$ and $g^{\prime}(z)=a \Rightarrow g^{\prime \prime}(z)=0$, we see that the zeros of $g$ and the zeros of $g^{\prime}-a$ coincide with finitely many exceptions, and that all zeros of $g^{\prime}-a$ are double with finitely many exceptions. Hence by Lemma $2.3, g$ has infinitely many zeros, and as $g$ is of finite order,

$$
\frac{g^{\prime}-a}{g^{2}}=Q e^{U}
$$

for some polynomials $Q(\not \equiv 0)$ and $U$. By (2.2), we have

$$
g^{\prime}=a+Q e^{U} g^{2}
$$

so that

$$
\begin{aligned}
g^{\prime \prime} & =\left(Q^{\prime}+Q U^{\prime}\right) e^{U} g^{2}+2 Q e^{U} g g^{\prime}=\left(Q^{\prime}+Q U^{\prime}\right) e^{U} g^{2}+2 Q e^{U} g\left(g^{2} Q e^{U}+a\right) \\
& =2 Q^{2} e^{2 U} g^{3}+\left(Q^{\prime}+Q U^{\prime}\right) e^{U} g^{2}+2 a Q e^{U} g .
\end{aligned}
$$

Thus by (2.1), we have

$$
2 Q^{2} e^{2 U} g^{2}+\left(Q^{\prime}+Q U^{\prime}\right) e^{U} g+2 a Q e^{U}=R
$$


Hence

$$
g(z)=0 \Rightarrow 2 a Q e^{U}=R(z) .
$$

Now differentiating the both sides of (2.5) yields that

$$
2\left(Q^{2} e^{2 U} g^{2}\right)^{\prime}+\left[\left(Q^{\prime}+Q U^{\prime}\right) e^{U}\right]^{\prime} g+\left(Q^{\prime}+Q U^{\prime}\right) e^{U} g^{\prime}+2 a\left(Q^{\prime}+Q U^{\prime}\right) e^{U}=R^{\prime},
$$

so that by $g(z)=0 \Rightarrow g^{\prime}(z)=a$, we get

$$
g(z)=0 \Rightarrow 3 a\left(Q^{\prime}+Q U^{\prime}\right) e^{U}=R^{\prime} .
$$

Hence, by (2.6) and (2.8), we see that

$$
g(z)=0 \Rightarrow \frac{3}{2}\left(\frac{Q^{\prime}}{Q}+U^{\prime}\right)=\frac{R^{\prime}}{R} .
$$

Since $g$ has infinitely many zeros, it follows that

$$
\frac{3}{2}\left(\frac{Q^{\prime}}{Q}+U^{\prime}\right) \equiv \frac{R^{\prime}}{R} .
$$

We claim that (2.10) is impossible. In fact, the residues of the right at the double poles of $R$ are -2 , while the residues of the left are not negative everywhere.

This contradiction shows that $g$ must be a rational function. This completes the proof of the lemma.

Lemma 2.6. Let $g$ be a nonconstant rational function with at most one pole, and let a be $a$ nonzero finite value. If $g(z)=0 \Rightarrow g^{\prime}(z)=a$ and $g^{\prime}(z)=a \Rightarrow g^{\prime \prime}(z)=0$, then $g(z)=a\left(z-z_{0}\right)$, where $z_{0}$ is a constant.

Proof. By Lemma 2.4, we only have to prove that $g$ is a polynomial. Suppose not, then by the condition, we may assume $g$ has only one pole 0 and it has multiplicity $m \geq 1$. Hence 0 is a double pole of $g^{\prime \prime} / g$. On the other hand, since $g(z)=0 \Rightarrow g^{\prime}(z)=a$ and $g^{\prime}(z)=a \Rightarrow g^{\prime \prime}(z)=0$, the zeros of $g$ are simple and $g(z)=0 \Rightarrow g^{\prime \prime}(z)=0$, so that $g^{\prime \prime} / g$ has no other poles, and hence $z^{2} g^{\prime \prime} / g$ is a polynomial. Since

$$
\frac{g^{\prime \prime}}{g}=\frac{g^{\prime \prime}}{g^{\prime}} \cdot \frac{g^{\prime}}{g}=O\left(\frac{1}{z}\right) \cdot O\left(\frac{1}{z}\right)=O\left(\frac{1}{z^{2}}\right)
$$

as $z \rightarrow \infty$, we then see that $z^{2} g^{\prime \prime} / g$ is a constant $c$. Obviously, $c \neq 0$.

Now write

$$
g(z)=z^{-m} \sum_{s=0}^{n} a_{s} z^{s}=\sum_{s=0}^{n} a_{s} z^{s-m},
$$


where $a_{s}$ are constants and $a_{0} \neq 0$. Then

$$
g^{\prime \prime}=\sum_{s=0}^{n}(s-m)(s-m-1) a_{s} z^{s-m-2} .
$$

Thus by $z^{2} g^{\prime \prime}=c g$, we get

$$
\sum_{s=0}^{n}(s-m)(s-m-1) a_{s} z^{s}=c \sum_{s=0}^{n} a_{s} z^{s} .
$$

So we can see $(s-m)(s-m-1) a_{s}=c a_{s}, s=0,1,2, \ldots, n$. It follows from $a_{0} \neq 0$ that $c=m(m+1)$, and hence $a_{s}=0, s=1,2, \ldots, 2 m, 2 m+2, \ldots, n$.

If $a_{2 m+1}=0$, we have $g(z)=a_{0} z^{-m}$. Obviously, the zeros of $g^{\prime}(z)-a$ are all simple, which contradicts $g^{\prime}(z)=a \Rightarrow g^{\prime \prime}(z)=0$.

If $a_{2 m+1} \neq 0$, we have $g(z)=a_{0} z^{-m}+a_{2 m+1} z^{m+1}$. Then

$$
g^{\prime}(z)-a=\frac{H(z)}{z^{m+1}}
$$

where

$$
H(z)=(m+1) a_{2 m+1} z^{2 m+1}-a z^{m+1}-m a_{0} .
$$

Since $g^{\prime}(z)=a \Rightarrow g^{\prime \prime}(z)=0$, we see that all zeros of $H(z)$ are multiple. Suppose $\zeta$ is a zero of $H(z)$, then

$$
H(\zeta)=(m+1) a_{2 m+1} \zeta^{2 m+1}-a \zeta^{m+1}-m a_{0}=0
$$

and

$$
H^{\prime}(\zeta)=(m+1)(2 m+1) a_{2 m+1} \zeta^{2 m}-(m+1) a \zeta^{m}=0
$$

We see that $\zeta \neq 0$, and by (2.14),

$$
\zeta^{m}=\frac{a}{(2 m+1) a_{2 m+1}}
$$

Then by (2.13),

$$
(m+1) a_{2 m+1}\left(\frac{a}{(2 m+1) a_{2 m+1}}\right)^{2} \zeta-\frac{a^{2}}{(2 m+1) a_{2 m+1}} \zeta-m a_{0}=0,
$$

hence we have

$$
\zeta=-\frac{(2 m+1)^{2} a_{0} a_{2 m+1}}{a^{2}} .
$$

This shows that $\zeta$ is a unique zero of $H(z)$, then we get

$$
H(z)=(m+1) a_{2 m+1}\left(z+\frac{(2 m+1)^{2} a_{0} a_{2 m+1}}{a^{2}}\right)^{2 m+1} .
$$

Comparing the coefficient of $z$ of (2.12) and (2.15), there is a contradiction.

The proof is completed. 


\section{Proofs of the main results}

Proof of Theorem 1.2. It suffices to show that $\mathscr{F}$ is normal at every point in $D$. Suppose that $\mathscr{F}$ is not normal at some point $z_{0} \in D$. By the condition, there exists $\delta>0$ such that every $f$ in $\mathscr{F}$ has at most one pole (simple or multiple) in $\Delta\left(z_{0}, \delta\right)$ and $\bar{\Delta}\left(z_{0}, \delta\right) \subset D$. Then by Lemma 2.1, we can find functions $f_{n} \in \mathscr{F}$, points $z_{n} \in \Delta\left(z_{0}, \delta\right)$ with $z_{n} \rightarrow z_{0}$, and positive numbers $\rho_{n} \rightarrow 0$ such that the sequence of functions $g_{n}(\zeta)=\rho_{n}^{-1} f_{n}\left(z_{n}+\rho_{n} \zeta\right)$ converges spherically locally uniformly on $\mathbb{C}$ to a nonconstant meromorphic function $g$ of finite order such that $g^{\sharp}(0)=(M+1)+1=M+2$, where $M=\max \left\{|a(z)|: z \in \bar{\Delta}\left(z_{0}, \delta\right)\right\}$.

We claim that

(i) $g$ has at most one pole;

(ii) $g(\zeta)=0 \Rightarrow g^{\prime}(\zeta)=a\left(z_{0}\right)$; and

(iii) $g^{\prime}(\zeta)=a\left(z_{0}\right) \Rightarrow g^{\prime \prime}(\zeta)=0$.

To prove (i), suppose that $g(\zeta)$ has two distinct poles $\zeta_{1}$ and $\zeta_{2}$. Then by Hurwitz's theorem, $g_{n}$ (for $n$ sufficiently large) has two distinct poles $\zeta_{n, 1}$ and $\zeta_{n, 2}$ such that $\zeta_{n, j} \rightarrow \zeta_{j},(j=$ 1,2). It follows from $g_{n}(\zeta)=\rho_{n}^{-1} f_{n}\left(z_{n}+\rho_{n} \zeta\right)$ that $f_{n}$ has has two distinct poles $w_{n, 1}=z_{n}+$ $\rho_{n} \zeta_{n, 1}$ and $w_{n, 2}=z_{n}+\rho_{n} \zeta_{n, 2}$. However, as both $w_{n, j} \rightarrow z_{0}$, this contradicts that every $f$ in $\mathscr{F}$ has at most one pole in $\Delta\left(z_{0}, \delta\right)$. The claim (i) is proved.

To prove (ii), let $\zeta_{0}$ be a zero of $g$. Then by Hurwitz's theorem, there exist points $\zeta_{n} \rightarrow \zeta_{0}$, such that $g_{n}\left(\zeta_{n}\right)=0$ (for $n$ sufficiently large), and hence $f_{n}\left(z_{n}+\rho_{n} \zeta_{n}\right)=0$. Thus by $f_{n}(\zeta)=0 \Rightarrow$ $f_{n}^{\prime}(\zeta)=a(\zeta)$, we have $g_{n}^{\prime}\left(\zeta_{n}\right)=f_{n}^{\prime}\left(z_{n}+\rho_{n} \zeta_{n}\right)=a\left(z_{n}+\rho_{n} \zeta_{n}\right)$. It follows that $g^{\prime}\left(\zeta_{0}\right)=\lim _{n \rightarrow \infty}$ $g_{n}^{\prime}\left(\zeta_{n}\right)=a\left(z_{0}\right)$. This proves (ii).

Next we prove (iii). Suppose that $g^{\prime}\left(\zeta_{0}\right)=a\left(z_{0}\right)$. Then $g\left(\zeta_{0}\right) \neq \infty$ and hence on some neighborhood $\Delta\left(\zeta_{0}\right)$ of $\zeta_{0}, g$ and $g_{n}$ (with $n$ sufficiently large) are holomorphic. Thus $g_{n}^{\prime}(\zeta)-$ $a\left(z_{n}+\rho_{n} \zeta\right) \rightarrow g^{\prime}(\zeta)-a\left(z_{0}\right)$ and $g_{n}^{\prime \prime}(\zeta) \rightarrow g^{\prime \prime}(\zeta)$ on $\Delta\left(\zeta_{0}\right)$. Hence by $g^{\prime}(\zeta) \not \equiv a\left(z_{0}\right)$ (for otherwise, $g(\zeta)=a\left(z_{0}\right) \zeta+c$ for some constant $c$ which contradicts that $\left.g^{\sharp}(0)=M+2>\left|a\left(z_{0}\right)\right|\right)$ and Hurwitz's theorem, there exist points $\zeta_{n} \rightarrow \zeta_{0}$ such that $g_{n}^{\prime}\left(\zeta_{n}\right)-a\left(z_{n}+\rho_{n} \zeta_{n}\right)=0$ (for $n$ sufficiently large). Since $g_{n}^{\prime}(\zeta)=f_{n}^{\prime}\left(z_{n}+\rho_{n} \zeta\right)$, we get $f_{n}^{\prime}\left(z_{n}+\rho_{n} \zeta_{n}\right)=a\left(z_{n}+\rho_{n} \zeta_{n}\right)$, and hence $\left|f_{n}^{\prime \prime}\left(z_{n}+\rho_{n} \zeta_{n}\right)\right| \leq h$ by the condition $f^{\prime}(z)=a(z) \Rightarrow\left|f^{\prime \prime}(z)\right| \leq h$. Since $g_{n}^{\prime \prime}(\zeta)=\rho_{n} f_{n}^{\prime \prime}\left(z_{n}+\rho_{n} \zeta\right)$, it follows that $\left|g_{n}^{\prime \prime}\left(\zeta_{n}\right)\right| \leq \rho_{n} h$, and hence $g^{\prime \prime}\left(\zeta_{0}\right)=0$ by $g_{n}^{\prime \prime}(\zeta) \rightarrow g^{\prime \prime}(\zeta)$. The claim (iii) is also proved.

Now by Lemma 2.5 and Lemma 2.6, $g$ must be of the form $g(\zeta)=a\left(z_{0}\right) \zeta+c$ for some constant $c$. This contradicts that $g^{\sharp}(0)=M+2>\left|a\left(z_{0}\right)\right|$.

Thus $\mathscr{F}$ is normal at every point in $D$ and hence on $D$. 


\section{References}

[1] J. M. Chang, M. L. Fang and L. Zalcman, Normal families of holomorphic functions, J. Math. Illinois, 48(2004), 319-337.

[2] J. M. Chang, A note on normality of meromorphic functions, Proc. Japan Acad., 83, Ser. A(2007), 60-62.

[3] J. Clunie and W. K. Hayman, The spherical derivative of integral and meromorphic functions, Comment. Math. Helv., 40(1966), 117-148.

[4] W. K. Hayman, Meromorphic Functions, Clarendon Press, Oxford,1964.

[5] K. L. Hiong, Sur les fonctions holomorphes dont les dérivées admettent une valeur exceptionnelle, Ann. Sci. École Norm. Sup. (3), 72(1955), 165-197.

[6] X. C. Pang and L. Zalcman, Normal families and shared values, Bull. London Math. Soc., 32(2000), 325-331.

[7] J. L. Schiff, Normal Families, Springer-Verlag, New York, 1993.

[8] W. Schwick, Sharing values and normality, Arch Math., 59(1993), 50-54.

[9] L. Yang, Value Distribution Theory, Spring-Verlag, Berlin, 1993.

Department of Mathematics, Changshu Institute of Technology, Changshu, Jiangsu 215500, P. R. China.

E-mail: xyliu_99@163.com 